\title{
Work, stress, and well-being of healthcare and human service professionals during outbreak of COVID-19
}

\author{
Kenny Kwong* \\ Graduate School of Social Work, Touro College, New York, USA
}

Received: June 7, 2021

Accepted: August 29, 2021

Online Published: September 9, 2021

DOI: $10.5430 /$ ijh.v7n2p15

URL: https://doi.org/10.5430/ijh.v7n2p15

\begin{abstract}
Objective: This study explored the level of occupational stress experienced by healthcare and human service professionals during COVID-19 pandemic and assessed if their personal characteristics, occupational stressors, job satisfaction, and their satisfaction with diversity of staff composition, salary, and compensation influenced their perceived stress, and their physical and psychological well-being.

Methods: A total of 227 healthcare and human service professionals participated in an online survey from March to August 2020. Participants provided background and job-related information and completed several measures to explore their perceived stress, job experiences, occupational stressors, as well as their physical and psychological problems. Bivariate analyses were used to assess the relationships between perceived stress, occupational stressors, satisfaction with job salary and compensation, satisfaction with diversity of staff composition, overall job satisfaction, and physical and psychological problems. Stepwise multiple regression analyses were performed to identify predictors of perceived stress and physical and psychological problems. Results: The study found a significant correlation between level of job satisfaction, satisfaction with diversity of staff composition, and satisfaction with job salary and compensation. Perceived stress was related positively with occupational stressors and physical and psychological problems, but negatively with overall job satisfaction, satisfaction with diversity of staff composition, and satisfaction with job salary and compensation. The findings found occupational stressors and being younger as significant predicators of perceived stress. Occupational stressors and female gender were significant predicators of experience of physical and psychological problems.

Conclusions: This study provides understanding on critical factors that have impacted healthcare and human service professionals' stress and wellbeing during outbreak of COVID-19 pandemic. These factors should be further investigated to inform public policy and interventions that mitigate health and mental health problems among these professionals during this and future outbreaks.
\end{abstract}

Key Words: COVID-19, Healthcare professionals, Work stress, Job satisfaction, Well-being, Career experience

\section{INTRODUCTION}

COVID-19 pandemic poses a significant challenge to healthcare and human service professionals who have direct contact with clients. Recent studies on work, stress, and mental health, focused on healthcare workers (HCWs) during outbreak of COVID-19 but very few studies explored the experi- ences of human service professionals. HCWs are vulnerable to emotional distress during the pandemic, given their risk of exposure to the coronavirus, intense work pressure, long working hours, having to serve a large volume of patients with multiple physical and psychological issues, and their concerns about caring and possibly infecting their family and loved ones. ${ }^{[1,2]}$

*Correspondence: Kenny Kwong; Email: kam.kwong@touro.edu; Address: Graduate School of Social Work, Touro College, New York, USA. 
Many recent studies on HCWs assessed the prevalence of depressive, anxiety, physical, and non-specific distress symptoms and identified key factors affecting health and mental health outcomes of HCWs. ${ }^{[3-9]}$ Salari et al. conducted a study to systematically review and assess the prevalence of stress, depression, and anxiety among frontline HCWs caring for COVID-19 patients and found the prevalence of depression, anxiety, and stress as $24.3 \%, 25.8 \%$, and $45 \%$ respectively. ${ }^{[3]}$ A systematic review and meta-analysis of 13 studies by Pappaa et al. showed that the pooled prevalence of anxiety, depression, and insomnia among HCWs in Singapore and China during outbreak of COVID-19 were $23.2 \%, 22.8 \%$, and $34.3 \%$, respectively. ${ }^{[4]}$ Sanghera et al. completed a systematic review to assess the effect of COVID19 on a range of psychological outcomes of hospital-based HCWs. ${ }^{[5]}$ The study found prevalence ranges of six psychological outcomes: acute stress reaction 5.2\%-32.9\%; posttraumatic stress disorder 7.4\%-37.4\%; anxiety $12.3 \%-35.6 \%$, depression $13.5 \%-44.7 \%$; insomnia $33.8 \%-36.1 \%$; and occupational burnout 3.1\%-43.0\%. ${ }^{[5]}$ A few studies reported physical or somatic symptoms experienced by HCWs. Shen et al., reported the prevalence of somatic symptoms, including poor appetite or indigestion (59\%) and fatigue (55\%). ${ }^{[7]}$ Pablo et al. completed a systematic review of published articles reporting somatic or physical symptoms in HCWs exposed to Severe Acute Respiratory Syndrome, Middle East Respiratory Syndrome and COVID-19. ${ }^{[6]}$ Of 115 articles reviewed, $75.9 \%$ of HCWs reported fever and $47.9 \%$ reported cough. The study also found that the self-reported rates of myalgias, chills, fatigue, headaches, dyspnoea, sore throat, nausea/vomiting, and diarrhea were $43.6 \%, 42.3 \%, 41.2 \%$, $34.6 \%, 31.2 \%, 25.3 \%, 22.2 \%$, and $18.8 \%$ respectively. ${ }^{[6]}$

Muller et al. completed a systematic review to identify risk factors attributing to psychological problems experienced by HCWs during outbreak of the pandemic and found that exposure to COVID-19 was the most common correlate of psychological problems, followed by female gender, and worry about being infected or infecting others. ${ }^{[8]}$ The study by Elbay et al. found that factors influencing mental health outcomes among frontline workers were being female, younger, single, and having less work experience. ${ }^{[9]}$ In the same study, factors found to correlate with higher scores of depression, anxiety, and stress among these workers were: increased weekly working hours, increased number of infected patients to serve, lower level of support from peers and supervisors, and decreased perceived level of practice competence on work-related tasks. ${ }^{[9]}$ Sanghera et al. also found that direct exposure to COVID-19 patients was the most common risk factor attributing to worse psychological outcomes. Those with pre-existing mental disorders and/or medical illnesses, as well as a lack of social support also reported negative outcomes. ${ }^{[5]}$ Healthcare workers, frontline staff, and nurses with fewer years of job experience and limited social support reported the worst outcomes. ${ }^{[5]}$

Most studies used quantitative methodology to assess the risk factors and outcomes experienced by HCWs during the COVID-19 pandemic. Only a few studies used qualitative research design to learn about HCWs' experiences and wellbeing during the COVID-19 pandemic. ${ }^{[10,11]}$ Benett et al. conducted a qualitative study of HCWs experiences of working with patients during the pandemic and found four specific themes: (1) the shock of the virus; (2) staff dedication and sacrifice; (3) collateral damage ranging from personal health concerns to the long-term impact on discharged patients; and (4) a hierarchy of power and inequality within the healthcare system. ${ }^{[10]}$ The study showed that while HCWs shared rewarding positive experiences, managing COVID-19 patients was a significant emotional challenge for them, resulting in strained relationships between frontline workers, their families, management and government. ${ }^{[10]}$ Hennein and Lowe used a hybrid inductive-abductive study design and thematic content analysis of HCWs' experiences and well-being during the COVID-19 pandemic and categorized HCWs' experiences within five ecological levels: individual level (self-care activities, personal health practices), interpersonal level (family functioning, social support, and well-being of people in their social circle), organization level (hospital's management, organizational support, team work, and patient care), community level (knowledge about the pandemic, community support of healthcare workers, exposure to media), and public policy level (leadership roles of health system and governments, government and healthcare system leadership, and shelter-in-place policy). ${ }^{[11]}$

Xiang et al. argued that most HCWs have received limited training on serving patients and community at large with significant mental health issues during outbreak of COVID19. ${ }^{12]}$ Saurabh and Ranjan surveyed doctors and nurses regarding their level of preparedness, concerns and perceived impact related to the pandemic outbreak. ${ }^{[13]}$ The study found that the majority of HCWs felt that their institution was ill prepared and did not have needed resources to cope with COVID 19 crisis. HCWs did not receive any training on the use of personal protection equipment or other infection control related training before the onset of the pandemic. ${ }^{[13]}$

\section{Methods}

\subsection{Research questions}

The present study explored the level of occupational stress experienced by healthcare and human service professionals during COVID-19 pandemic and the methods they used to 
cope with occupational stress. The study also assessed if their personal characteristics, work-related problems, job satisfaction, and their satisfaction with diversity of staff composition, salary, and compensation influenced their perceived stress, and their physical and psychological well-being. Specifically, the study addressed three major questions:

(1) What are the job experiences of healthcare and human service professionals, i.e., level of satisfaction with the diversity of staff composition, job salary and compensation, and overall job satisfaction?

(2) What is the level of perceived stress experienced by healthcare and human service professionals working in New York City during COVID-19 pandemic? How they do they cope with occupational stress? In what ways do their personal characteristics, occupational stressors, their overall job satisfaction, and their satisfaction with staff composition, salary, and compensation influence their perceived stress?

(3) Which occupational stressors, personal characteristics, and other factors influence their physical and psychological well-being?

\subsection{Study procedures}

This study used an online cross-sectional survey research design to answer the research questions. Convenient and snowball sampling design was used to recruit healthcare and human service professionals who provided direct services in different sectors such as health, mental health, child welfare, family services, schools, and specialized programs in New York City, an epicenter of the COVID-19 outbreak in the United States during spring 2020. The survey was conducted from March to August 2020. An invitation email to solicit participation was sent by students and faculty of a graduate school of social work to their friends and colleagues who work at healthcare or human service agencies in NYC. Participants were asked to share the study with their friends and colleagues to invite them to complete the survey. The study used Qualtrics Survey Software to create a survey link. A consent letter with the survey link was shared with all potential participants to let them know that the objective of the study was to learn about their work, stress, and well-being and if COVID-19 pandemic had impacted on their work, stress, and well-being. They were instructed to share their background and job-related information and answer several scales to assess their occupational stressors and job experiences, as well as their physical and psychological problems. These scales include Perceived Stress Scale, ${ }^{[14]}$ Occupational Stressors, ${ }^{[15]}$ and Psychological and Physical Problems. ${ }^{[15]}$ Participants were told that it would take 5-7 minutes to complete the survey and that their completion of Published by Sciedu Press the study was entirely voluntary. The study was approved by the Institutional Review Board of the researcher's affiliated institution.

\subsection{Participants}

Two hundred and twenty-seven healthcare and human service professionals completed the survey. Their average age was 38.6 (range 20-70). Approximately $70 \%$ were females, $28.4 \%$ were male, and $1.3 \%$ were transgender. The majority identified themselves as Jewish Americans (38.3\%) followed by African Americans (28.2\%), White (16.3\%), Latino/Hispanic American (8.4\%), Asian American/Pacific Islanders (4\%), American Indians (.9\%), and multi-racial (.9\%). About half (52.4\%) were married; $44.5 \%$ had children under 18 living at home with them and $55.5 \%$ did not. The majority worked full-time $(80.4 \%)$. Majority of participants had either a master's degree $(50.2 \%)$, a bachelor's degree $(31.7 \%)$, or a post-graduate degree $(7.5 \%)$.

\subsection{Measures}

\subsubsection{Background and job-related characteristics}

Personal characteristics such as gender, age, marital status, level of educational attainment, race/ethnicity, whether they have children under age 18, work status, work title, and job tenure, were assessed. Participants were also asked to indicate in a 4-point Likert scale ranging from (1) "very satisfied" to (4) "very unsatisfied" to assess their satisfaction with their job salary and compensation, their satisfaction with the diversity of staff composition in their agency that met the needs of the population their agency targeted to serve, and their overall job satisfaction.

\subsubsection{Perceived stress scale}

The 10-item Perceived Stress Scale was used to assess how participants felt about different work situations and to what extent they appraised their life situations as stressful. ${ }^{[14]}$ It is a popular psychological measure to assess the perception of stress. The internal consistency reliability was .88 for this study sample.

\subsubsection{Occupational stressors}

The Occupational Stressors scale comprised of 18 job-related stressors often experienced by human service professionals. ${ }^{[15]}$ Participants were asked to indicate how often they experienced these problems in a 4-point Likert scale ranging from (4) "very often" to (1) "never/rarely." Factor analysis was conducted by grouping correlated variables into a few factors, making analyses more manageable. Reliability analyses were conducted with 18 variables and one variable was removed, resulting in 4 major factors: (1) job nature, quality, and support; (2) workload and balance of life/work commitment; (3) salary and compensation; and (4) challenging 
client and staff situations. The internal consistency measure yielded a high reliability of .88 for the sample.

\subsubsection{Physical and Psychological Problems}

This study adapted the Job-Related Health Problems Scale ${ }^{[15]}$ and included a list of 14 psychological and physical problems. Participants were asked to indicate in a 4-point Likert scale how frequent they had these problems ranging from (4) "very often" to (1) "never/rarely." Factor analysis was done by grouping correlated variables into a few factors to facilitate data analyses. Reliability analyses were performed, and 5 variables were removed from the list. The Somatic/Physical Factor includes 4 symptoms - back and neck pain, lack of energy or fatigue, stomach/digestive problems, and difficulties in sleeping. The Psychological Factor includes 5 symptoms - feeling tense, nervous, anxious, or fidgety; feeling powerless; feeling aggressive and irritable; depression; and difficulties in concentration. The internal consistency reliability of the 2-factor scale was .86 .

\subsubsection{Stress management}

The NASW Membership Workforce Study listed a range of methods social workers employed to cope with job-related problems and stressors. ${ }^{[16]}$ The present study included some of these methods and added a few more that might be employed by healthcare and human service professionals to manage their stress. Participants were asked if they have used any of the 10 methods that helped them manage occupational stressors and regain a sense of control in their life. These 10 methods include: mind, body, and spirit ap- proach, psychological counseling or therapy, seeking support through a social or professional network; physical activity, stress management, absenteeism, tobacco use, alcohol use, use of prescription drug, and other activities that make their life enjoyable.

\subsection{Statistical analysis}

All the data analyses were performed with the SPSS, Version 26. Descriptive statistics include means and standard deviations for all ratio/interval level variables and raw frequency and frequency percentages for all nominal/ordinal level variables. Factor analyses were performed for two specific measures - Occupational Stressors and Physical and Psychological Problems. Reliability measures were performed to assess the internal consistency of major measures. Bivariate analyses were used to determine correlation and linear association between variables. Finally multiple regression analyses were used to identify significant predicators of major outcome variables.

\section{Results}

\subsection{Bivariate statistics}

Table 1 below showed the correlations between perceived stress, occupational stressors, physical and psychological problems, satisfaction with job salary and compensation, satisfaction with diversity of staff composition, overall job satisfaction. Means and standard deviations of these major study variables were displayed.

Table 1. Means, standard deviations, and correlations among study variables

\begin{tabular}{|c|c|c|c|c|c|c|c|c|}
\hline Variable & Mean & SD & 1 & 2 & 3 & 4 & 5 & 6 \\
\hline 1. Perceived Stress & 27.35 & 6.70 & 1 & & & & & \\
\hline 2. Physical and Psychological Problems & 28.16 & 5.48 & $.58^{* *}$ & 1 & & & & \\
\hline 3. Occupational Stressors & 51.12 & 9.28 & $.39^{* *}$ & $.53^{* *}$ & 1 & & & \\
\hline 4. Job Salary Satisfaction & 2.26 & .92 & $-.24^{* *}$ & $-.27^{* *}$ & $-.44^{* *}$ & 1 & & \\
\hline 5. Staff Diversity Satisfaction & 2.15 & .92 & $-.24^{* *}$ & $-.21^{*}$ & $-.34^{* *}$ & $.34^{* *}$ & 1 & \\
\hline 6. Overall Job Satisfaction & 1.86 & .88 & $-.34^{* *}$ & $-.38^{* *}$ & $-.49^{* *}$ & $.59^{* *}$ & $.39^{* *}$ & 1 \\
\hline
\end{tabular}

Among three variables of job-related experiences, their overall job satisfaction was correlated strongly with their satisfaction with diversity of staff composition $(r=.39, p<.01)$ and their satisfaction with job salary and compensation given their responsibility $(r=.59, p<.01)$. Perceived stress was related positively with occupational stressors $(r=39, p<$ $.01)$ and physical and psychological problems $(r=.58, p<$ $.01)$, but negatively with overall job satisfaction $(r=-.34, p<$
$.01)$, satisfaction with diversity of staff composition $(r=-.24$, $p<.01)$, and satisfaction with job salary and compensation $(r=-.24, p<.01)$. Physical and psychological problems was found to associate positively with occupational stressors $(r=$ $.53, p<.01)$ but negatively with overall job satisfaction $(r$ $=-.38, p<.01)$, satisfaction with diversity of staff composition $(r=-.21, p<.05)$, and satisfaction with job salary and compensation $(r=-.27, p<.01)$. 


\subsection{Management and predictors of occupational stress}

Two hundred and twenty-seven (227) participants working in NYC during COVID-19 pandemic shared the methods they used in managing their occupational stress. For healthcare and human service professionals in this sample, engaging activities that made their life enjoyable was the most common strategy of managing stress $(\mathrm{N}=127,56 \%)$, followed by physical activity $(\mathrm{N}=123,54 \%)$, mind, body, and spirit approach $(\mathrm{N}=111,49 \%)$, and seeking help through social networks or professional groups $(\mathrm{N}=88,39 \%)$. Some sought professional counseling or therapy $(\mathrm{N}=50,22 \%)$ or practice stress management activities $(\mathrm{N}=62,27 \%)$. Few used prescription drug $(\mathrm{N}=31,14 \%)$, absenteeism $(\mathrm{N}=22,10 \%)$, alcohol use $(\mathrm{N}=39,17 \%)$, or tobacco use $(\mathrm{N}=15,7 \%)$ to manage occupational stress.

To identify factors that best predict perceived stress of this sample, a stepwise multiple regression analysis was performed. Six possible factors including gender, age, occupational stressors, job satisfaction, satisfaction with job salary and compensation, and satisfaction with diversity of staff composition were entered. These factors were chosen because they all demonstrated significant bivariate relationships with perceived stress. The findings identified two significant factors $(F=21.09 ; p<.05)$. With a beta of $.45(p<.05)$, occupational stressors were found to be the strongest factor, explaining $20 \%$ of the variance in perceived stress (see Table $2)$. Age was found as another important factor $(\beta=-.23 ; p<$
$.05)$ explaining $5.3 \%$ of the variance. The findings showed that higher level of perceived stress was a result of higher level of occupational stressors and being younger. Overall, the model accounted for $25 \%$ of the variance in perceived stress $(R=.50)$.

\subsection{Factors attributing to physical and psychological problems}

To identify factors that could best predict physical and psychological problems experienced by these workers, another stepwise multiple regression analysis was performed. Eight possible factors including gender, education, having children under 18, work schedule, occupational stressors, satisfaction with job salary and compensation, satisfaction with diversity of staff composition, and job satisfaction were entered. These factors were chosen because they all demonstrated significant bivariate relationships with physical and psychological problems. The findings identified two significant factors $(F=35.13 ; p<.05)$. With a beta of $.59(p<.05)$, occupational stressors were found to be the strongest factor, explaining $36 \%$ of the variance in physical and psychological problems (see Table 3 ). The second factor was gender $(\beta=$ $-.21 ; p<.05)$ accounting for $4.5 \%$ of the variance in physical and psychological problems. The study found that higher physical and psychological problems was a result of higher level of occupational stressors and being female. Overall, the regression model accounted for almost $40 \%$ of the variance in physical and psychological problems $(R=.64)$.

Table 2. Predicting factors of perceived stress

\begin{tabular}{llllllll}
\hline Factor & $\boldsymbol{R}$ & $\boldsymbol{R}^{2}$ & $\boldsymbol{\beta}$ & $\boldsymbol{t}$ & $\boldsymbol{p}$ & $\boldsymbol{F}$ & $\boldsymbol{p}$ \\
\hline Occupational Stressors & .45 & .20 & .45 & 5.83 & .000 & 31.42 & .000 \\
Age & .50 & .25 & -.23 & -2.97 & .004 & 21.09 & .000 \\
\hline
\end{tabular}

Table 3. Predicting factors of physical and psychological problems

\begin{tabular}{llllllll}
\hline Factor & $\boldsymbol{R}$ & $\boldsymbol{R}^{2}$ & $\boldsymbol{\beta}$ & $\boldsymbol{t}$ & $\boldsymbol{p}$ & $\boldsymbol{F}$ & $\boldsymbol{p}$ \\
\hline Occupational Stressors & .60 & .36 & .59 & 8.57 & .000 & 70.20 & .000 \\
Gender & .64 & .40 & -.21 & -3.05 & .003 & 42.08 & .000 \\
\hline
\end{tabular}

\section{Discussion}

\subsection{Major findings and implications}

Healthcare workers are at particular risk of suffering from mental health problems when they work in emergency care, critical or intensive care settings. ${ }^{[17]}$ They are vulnerable to occupational stress due to unprecedented circumstances such as working with dying and seriously ill patients, increased workload, lack of support and resources, challenges in providing quality care, organizational conflicts and problems, and are at high risk to suffer from the impact of work-related problems and situations during COVID-19 pandemic. ${ }^{[18]}$ The findings of this study were consistent with the literature, ${ }^{[9,18,19]}$ and showed that occupational stressors (as measured in this study by life/work balance, workload, job nature and quality, salary and compensation, and challenging client and staff situations) were significant predictors of both perceived stress and physical and psychological problems for these workers. The results added to the literature and found that overall job satisfaction, satisfaction with diversity of 
staff composition that met the needs of the population the agency targeted to serve, and satisfaction with job salary and compensation, as significant correlates of perceived stress. Additionally, the findings revealed being younger as a significant predicator of perceived stress, and female gender as a significant predicator of experience of physical and psychological problems. These findings were consistent with the literature ${ }^{[8,9]}$ and these factors as well as other possible risk factors should be investigated more extensively in future studies.

Occupational stress may compromise professional efficacy, causing decreased attention, reducing decision-making abilities, and negatively impacting the worker's ability to establish a working relationship with their clients. ${ }^{[18]}$ It is imperative for healthcare and human service workers to develop effective stress coping strategies and maintain optimal mental health on a regular basis, particularly during a pandemic. Notably for healthcare and human service professionals in this sample, engaging activities that made their life enjoyable was the most common approach in managing stress, followed by mind, body, and spirit approach, physical activity, and seeking help through social networks or professional groups. Carmassi et al. argue that when planning effective intervention strategies at an organizational level, it is critical to consider resilience factors such as effective work environment, job training and support, and effective stress coping methods, to promote the resilience and reduce the risk of adverse physical and psychological outcomes among HCWs. ${ }^{[19]}$

\subsection{Study limitations}

This study has several limitations. The study sample was diverse in gender, marital status, race/ethnicity, education level, having children under 18 living with them, and work schedule. However, the sample may not represent the large population of healthcare and human service professionals because of sampling bias due to snowball and convenience sampling design. Thus, study results were weak in generalizability. The analyses of findings cannot assert causality in a cross-sectional survey design study; thus, the study cannot compare the pre-pandemic to pandemic phases to show if the COVID-19 pandemic had increased level of occupational stress among healthcare and human service professionals. There were likely recall bias when participants self-report their perception and experiences. The extent of such biases may depend on how they perceived their career and job environment and their job tenure. Participants were reassured about the anonymity nature of the study and encouraged to share their opinion, perceptions, and experiences honestly.

\section{Conclusions}

Healthcare and human service professionals are at high risk of experiencing occupational stressors. It is important for them to strengthen their abilities and skills in managing jobrelated problems and stressors, maintain a delicate balance between work and personal life, make connection to obtain professional help, seek family and social support, and regain a sense of control and efficacy over their work environments. ${ }^{[15,20]}$ More studies on the sources and extent of occupational stressors, perceived stress, and physical and psychological problems among healthcare and human service workers are much needed to help design interventions and career development for these professionals to learn how to cope with occupational stress and enhance their own physical and mental well-being and self-care. ${ }^{[21]}$ The World Health Organization recognizes the occupational risk to HCWs may attribute to worsen mental health outcomes such as posttraumatic stress, depression, and burnout. ${ }^{[22]}$ Interventions and services are much needed to alleviate stress and anxiety among this vulnerable population. The Centers for Disease Control and Prevention also provide recommendations for frontline health workers to reduce their occupational stress such as increasing awareness of physical and psychological symptoms, taking breaks from work and media coverage, seeking professional help, and engaging in self-care. ${ }^{[23]}$ This study contributed to a growing body of literature ${ }^{[8-11,17-19]}$ that assessed the nature and sources of psychological stress experienced by healthcare and human service professionals, their stress coping methods, job-related experiences, and psychological and physical health. The findings of this study provide understanding of critical factors that have impacted their psychological stress and well-being during the COVID19 pandemic. These factors should be further explored to inform public policy and interventions that mitigate health and mental health problems among healthcare and human service professionals during this and future outbreaks.

\section{ACKNOWLEDGEMENTS}

The author wants to thank all the students and faculty colleagues who helped invite their agency colleagues and friends to participate in the study. The study was supported by Touro College Graduate School of Social Work.

\section{CONFLiCTS OF INTEREST Disclosure}

The author declares no conflicts of interest. 


\section{REFERENCES}

[1] Banerjee D, Vijayakumar HG, Rao TS. "Watching the watchmen:" Mental health needs and solutions for the health-care workers during the coronavirus disease 2019 pandemic. Int J Health Allied Sci. 2020; 9(Suppl S1): 51-4.

[2] Pfefferbaum B, North CS. Mental health and the Covid-19 pandemic. N Engl J Med. 2020; 383(6): 510-2. PMid:32283003. https://doi.org/10.1056/NEJMp2008017

[3] Salari N, Khazaie H, Hosseinian-Far A, et al. The prevalence of stress, anxiety and depression within front-line healthcare workers caring for COVID-19 patients: a systematic review and metaregression. Hum Resour Health. 2020; 18: 100. PMid:33334335. https://doi.org/10.1186/s12960-020-00544-1

[4] Pappaa S, Ntellac V, Giannakasc T, et al. Prevalence of depression, anxiety, and insomnia among healthcare workers during the COVID19 pandemic: A systematic review and meta-analysis. Brain Behav Immun. 2020

[5] Sanghera J, Pattani N, Hashmi Y, et al. The impact of SARS-CoV-2 on the mental health of healthcare workers in a hospital setting - a systematic review. J Occup Health. 2020; 62(1). PMid:33131192. https://doi.org/10.1002/1348-9585.12175

[6] Pablo GSD, Vaquerizo-Serrano J, Catalan A, et al. Impact of coronavirus syndromes on physical and mental health of health care workers: systematic review and meta-analysis. J Affect Disord. 2020; 275: 48-57. PMid:32658823. https ://doi .org/10.1016/j.ja d. 2020.06 .022

[7] Shen X, Zou X, Zhong X, et al. Psychological stress of ICU nurses in the time of COVID-19. Crit. Care. 2020; 24(1): 200. PMid:32375848. https://doi.org/10.1186/s13054-020-02926-2

[8] Muller AE, Hafstad EV, Himmels JPW, et al. The mental health impact of the covid-19 pandemic on healthcare workers, and interventions to help them: a rapid systematic review. Psychiatry Res. 2020; 293: 113441. PMid:32898840. https ://doi.org/10.101 $6 / j \cdot$ psychres. 2020.113441

[9] Elbay RY, Kurtulmuş A, Arpacioğlu S, et al. Depression, anxiety, stress levels of physicians and associated factors in Covid-19 pandemics. Psychiatry Res. 2020; 290: 113130. PMid:32497969. https ://doi.org/10.1016/j.psychres.2020.113130

[10] Bennett P, Noble S, Johnston S, et al. COVID-19 confessions: a qualitative exploration of healthcare workers experiences of working with COVID-19. BMJ Open. 2020; 10(12). PMid:33328264. https://doi.org/10.1136/bmjopen-2020-043949

[11] Hennein R, Lowe S. A hybrid inductive-abductive analysis of health workers' experiences and wellbeing during the COVID-19 pan- demic in the United States. Plos One. 2020; 15(10). PMid:33104711. https://doi.org/10.1371/journal.pone.0240646

[12] Xiang YT, Yang Y, Li W, et al. Timely mental health care for the 2019 novel coronavirus outbreak is urgently needed. The Lancet Psychiatry. 2020; 7(3): 228-9. https://doi .org/10.1016/S221 5-0366 (20) 30046-8

[13] Saurabh K, Ranjan S. Preparedness, perceived impact and concerns of health care workers in a teaching hospital during coronavirus disease 2019 (COVID-19). J Family Med Prim Care. 2020; 9(8): 4247-51. PMid:33110840.https://doi.org/10.4103/jfmpc.jfmpc_79 9_20

[14] Cohen S, Kamarck T, Mermelstein R. A global measure of perceived stress. J Health Soc Behav. 1983; 24(4): 385. PMid:6668417. https://doi.org/10.2307/2136404

[15] Kwong K. Assessing secondary trauma, compassion satisfaction, and burnout - implications for professional education for AsianAmerican social workers. Int J Higher Educ. 2018; 7(5): 75. https : //doi.org/10.5430/ijhe.v7n5p75

[16] Arrington P. Stress at work: How do social workers cope? NASW Membership Workforce Study. Washington, DC: National Association of Social Workers; 2008.

[17] Cullen W, Gulati G, Kelly BD. Mental health in the COVID-19 pandemic. QJM. 2020; 113(5): 311-2. PMid:32227218. https : //doi.org/10.1093/qjmed/hcaa110

[18] Irfan M, Naeem F, Afridi M, et al. Prevention of occupational stress in health-care workers during COVID-19 pandemic. Indian J Psychiatry. 2020; 62(9): 495-7. PMid:33227042. https://doi.org/10 $.4103 /$ psychiatry. IndianJPsychiatry_844_20

[19] Carmassi C, Foghi C, Delloste V, et al. PTSD symptoms in healthcare workers facing the three coronavirus outbreaks: what can we expect after the COVID-19 pandemic. Psychiatry Res. 2020; 292: 113312 PMid:32717711. https://doi.org/10.1016/j.psychres. 202 0.113312

[20] Shoji K, Bock J, Cieslak R, et al. Cultivating secondary traumatic growth among healthcare workers: the role of social support and self-efficacy. J Clin Psychol. 2014; 70(9): 831-46. PMid:24420964. https://doi.org/10.1002/jclp. 22070

[21] Kwong K. Understanding work-related stress and practice of professional self-care - an innovative pedagogical approach. Int J Higher Educ. 2016; 5(4): 41-51. https://doi.org/10.5430/ijhe.v5n $4 \mathrm{p} 41$

[22] World Health Organization. Mental health considerations during COVID-19 outbreak. Geneva, World Health Organization, 2020.

[23] Centers for Disease Control and Prevention. Manage anxiety \& stress. Atlanta, GA, Centers for Disease Control and Prevention, 2020. 